\title{
EVALUACIÓN DEL ESTADO DE SALUD MENTAL DE LOS CUIDADORES DE PACIENTES CON ENFERMEDADES CRÓNICAS NO TRANSMISIBLES DE LA COMUNIDAD DE EL LLANO, PROVINCIA PERAVIA, REPÚBLICA DOMINICANA, DURANTE EL PERÍODO NOVIEMBRE-DICIEMBRE 2019
}

\section{Evaluation of mental health status in caregivers of patients with chronic non-communicable diseases of the community of El Llano, Peravia Province, Dominican Republic, during the period November-December 2019}

\section{Skarlet Ferreras ${ }^{a}$, Luz Maríab , Julissa Matosc ${ }^{c}$ Emilton López ${ }^{\mathrm{d}}$ y Penélope Brito Lee}

Recibido: 23 de mayo, $2021 \bullet$ Aprobado: 23 de agosto, 2021

Cómo citar: Ferreras S, María L, Matos J, López E, Brito Lee P. Evaluación del estado de salud mental de los cuidadores de pacientes con enfermedades crónicas no transmisibles de la comunidad de El Llano, provincia Peravia, República Dominicana, durante el período noviembre-diciembre 2019. cysa [Internet]. [citado 22 de febrero de 2022];6(1):87-93. Disponible en: https://revistas.intec.edu.do/ index.php/cisa/article/view/2400

\section{Resumen}

Introducción: el Síndrome del cuidador (CIE-11 QF27) se define como una respuesta inadecuada al estrés emocional crónico, que no aparece exclusivamente en los cuidadores y cuyas principales características son el agotamiento físico y/o psicológico ${ }^{1}$. Es una entidad de gran prevalencia en la actualidad, y ello se debe tanto al aumento de la esperanza de vida como al auge de las enfermedades crónicas no transmisibles, estas últimas incapacitando a la población y aumentando el número de cuidadores formales e informales, que surgen para atender las necesidades de los afectados ${ }^{2}$.

Objetivos: evaluar el estado de salud mental en cuidadores de pacientes con enfermedades crónicas no transmisibles de la comunidad de El Llano, provincia de Peravia, República Dominicana.

Metodología: estudio transversal, prospectivo, observacional y analítico. Se seleccionó una muestra de 115

\footnotetext{
a Instituto Tecnológico de Santo Domingo, (INTEC), Santo Domingo, República Dominicana. ORCID: 0000-0003-2639-5795

Correo-e:1067183@est.intec.edu.do

b Instituto Tecnológico de Santo Domingo, (INTEC).

ORCID: https://orcid.org/0000-0002-8861-7181

Correo-e: 1067266@est.intec.edu.do
}

\begin{abstract} emerge to meet the needs of those affected ${ }^{2}$. nican Republic.

\footnotetext{
${ }^{\mathrm{c}}$ Instituto Tecnológico de Santo Domingo, (INTEC). ORCID: https://orcid.org/0000-0002-1334-545X Correo-e: 1064857@est.intec.edu.do

d Asesor. Correo-e: emilton.lopez@intec.edu.do

e Asesor. Correo-e: penelopebrito_lee@hotmail.com
}

Introduction: The Caregiver Stress Syndrome (ICD-11 QF27) is defined as an inadequate response to chronic emotional stress, which does not appear exclusively in caregivers and whose main features are physical and/or psychological exhaustion ${ }^{1}$. It is an entity of great prevalence nowadays, and this is due both to the increase in life expectancy and to the rise of chronic non-communicable diseases, the latter disabling the population and increasing the number of formal and informal caregivers, who

Objectives: Evaluate the mental health status in caregivers of patients with chronic non-communicable diseases of the community of El Llano, Peravia province, Domi- 
personas, con un nivel de confianza del $95 \%$ y un margen de error del $8 \%$, a las que se les aplicaron los cuestionarios. Las variables del cuestionario incluyeron edad, sexo, fecha de la información recolectada, así como los cuestionarios estandarizados de Goldberg para depresión y ansiedad en atención primaria, Cuestionario para la sobrecarga de Zarit y fragmentos del Cuestionario para cuidadores primarios. Además, se incluyó un formulario para adquirir el consentimiento informado.

Resultados: se encontró que el $63 \%$ de los cuidadores podía sufrir depresión, el $52 \%$ podía sufrir ansiedad y el $71 \%$ de los cuidadores no presentaba sobrecarga. Sin embargo, la sobrecarga no es suficiente para explicar el comportamiento de depresión $(\mathrm{r} 2=-0,27)$ o ansiedad $(\mathrm{r} 2=-0,14)$, ya que ambos demostraron una correlación positiva muy baja. Se demostró que un $76 \%$, correspondiente a 87 cuidadores, eran del sexo femenino. También la edad fue una variable importante, e $45 \%$ de los cuidadores se encontraron entre las edades de 41-60 años.

Conclusiones: la sobrecarga presentada por los encuestados no fue suficiente para explicar los niveles de depreión y ansiedad por lo tanto, se debe conrlacion más variables.

Palabras clave: salud mental; enfermedades crónicas no transmisibles; síndrome del cuidador; psiquiatría; atención primaria

\section{Introducción}

El síndrome del cuidador (CIE-11 QF27) se define como una respuesta inadecuada a un estrés emocional crónico, que no aparece exclusivamente en cuidadores y cuyos rasgos principales son el agotamiento físico y/o psicológico ${ }^{1}$. Es una entidad de gran prevalencia hoy en día, y esto se debe tanto al aumento de la expectativa de vida como al auge de enfermedades crónicas no transmisibles ${ }^{2}$. Estas últimas son la principal causa de muerte y discapacidad prematura en la gran mayoría de los países de América Latina y el Caribe, lo cual aumenta la cantidad de cuidadores formales e informales que surgen para suplir las necesidades de los afectados ${ }^{3,4}$

El cuidador de una persona con enfermedad crónica ve afectada su calidad de vida y comprometido su
Methodology: Cross-sectional, prospective, observational, and analytical study. A sample of 115 people was selected, with a 95\% confidence level and 8\% margin of error, to which the questionnaires were applied. The questionnaire variables included age, gender, date of collected information, as well as Goldberg's standardized questionnaires for depression and anxiety in primary care, Zarit Burden Interview, and fragments of the Primary Caregivers Questionnaire. Additionally, a form to acquire informed consent was included.

Results: It was found that $63 \%$ of caregivers could suffer from depression, 52\% could suffer from anxiety, and 71\% of caregivers did not present burden. Meanwhile, the overload is not enough to explain the behavior of depression $(\mathrm{r} 2=-0.27)$, or anxiety $(\mathrm{r} 2=-0.14)$ since both demonstrated a very low positive correlation. It was shown that $76 \%$ corresponding to 87 caregivers were female. Age was also an important caregivers were between ages of 41-60 years.

Conclusions: The overload presented by the respondents was not sufficient to explain the levels of depression and anxiety, hence should be correlated with other variables.

Keywords: Mental health; chronic non-communicable diseases; Caregiver Stress Syndrome; Psychiatry; Primary care.

núcleo familiar5. La calidad de vida es un concepto muy importante que involucra diversas variables relacionadas con el bienestar humano, como lo son: la salud física y mental, el nivel de independencia, las relaciones sociales, las creencias personales y la relación con las características sobresalientes del entorno ${ }^{5}$. La afectación de todas estas variables que definen la calidad de vida promueven la aparición de un conjunto de problemas físicos, mentales y socioeconómicos a los cuales se les denomina carga y cuya persistencia es la causa principal del desarrollo del síndrome ${ }^{1}$.

La salud mental, según la Organización Mundial de la Salud (OMS), es un estado de completo bienestar psicológico, y no únicamente la ausencia de trastornos mentales ${ }^{6}$. En el síndrome del cuidador se evidencian grandes alteraciones a la salud mental del afectado, que se manifiestan en el cambio de actitud, irritabilidad, fatiga, y hasta en el desarrollo de trastornos como la ansiedad y la depresión ${ }^{7}$, por lo que evaluar a los cuidadores de pacientes con enfermedades crónicas no transmisibles representa el objetivo principal de la presente investigación, para conocer su salud mental y crear soluciones contra las limitantes que estos enfrentan.

\section{Material y métodos}

La presente investigación fue de tipo transversal, prospectivo, observacional y analítica. La muestra fue obtenida con la fórmula para variable cualitativa con población conocida $n=\frac{N Z_{2}^{2} p q}{d^{2}(N-1)+z^{2} 2 q}$, para una población de 523 pacientes con enfermedades crónicas no transmisibles, un $\mathrm{z}=1.96$ (Intervalo de Confianza de $95 \%$ ), una prevalencia de enfermedades crónicas no transmisibles de $\mathrm{p}=45 \%{ }^{8}$ y una $\mathrm{d}=8 \%$, se obtuvo un total de 115 sujetos.

El instrumento de recolección de datos fue elaborado por los autores de la presente investigación, y fue utilizado para analizar el estado de salud mental de los cuidadores principales de pacientes con enfermedades crónicas no transmisibles. Las variables del cuestionario contemplaron edad, sexo, la fecha de la recolección de la información, además de incluir los cuestionarios estandarizados de Goldberg para depresión y ansiedad en atención primaria, el test de Zarit para conocer el nivel de sobrecarga del cuidador y fragmentos de la Encuesta de Discapacidad, Autonomía personal y situaciones de Dependencia: Cuestionario de Cuidadores/as principales. Estos cuestionarios estandarizados, aún creados para realizarse en atención primaria, fueron interpretados por un especialista en salud mental.

El cuestionario utilizado para la evaluación de la sobrecarga fue el Test de sobrecarga de Zarit. Este contempla una sensibilidad del $100 \%$ y una especificidad del $90.5 \%$ 9,10.
La escala de Goldberg, utilizada para predecir depresión y ansiedad en atención primaria, cuenta con una sensibilidad de $83.1 \%$ y una especificidad de $81.8 \%{ }^{11,12}$.

La Encuesta de Discapacidad, Autonomía personal y situaciones de Dependencia: Cuestionario de Cuidadores/as principales, elaborado por el Instituto Nacional de Estadística de España tiene una sensibilidad de un $89.4 \%$ y una especificidad de un $92.6 \%{ }^{13}$.

Antes de la aplicación de cada cuestionario se procedió a leer y firmar el consentimiento informado con los pacientes.

Para recopilación de la base teórica y antecedentes sobre el tema se utilizaron motores de búsqueda como Cochrane Library, UpToDate, Medscape, Google Scholar, Merck Manuals y el Manual del Residente en Geriatría por la Sociedad Espańola de Geriatría y Gerontología.

Los datos obtenidos fueron analizados estadísticamente mediante el programa Microsoft Excel Versión Office 2017.

\section{Resultados}

Tabla 1. Niveles de depresión y ansiedad. $(\mathrm{n}=115)$

\begin{tabular}{|l|c|c|}
\hline & $\begin{array}{c}\text { Casos } \\
\text { probables }\end{array}$ & $\begin{array}{c}\text { Ausencia de } \\
\text { signos }\end{array}$ \\
\hline Depresión & $73(63 \%)$ & $42(37 \%)$ \\
\hline Ansiedad & $60(52 \%)$ & $55(48 \%)$ \\
\hline
\end{tabular}

Fuente: Ferreras, María, Matos. Internado Medicina Social, noviembre-diciembre 2019. 
Según la prueba de Goldberg, para la subescala de depresión una cantidad mayor o igual a dos (2) respuestas positivas indicaría un $50 \%$ de probabilidad de una alteración clínicamente significativa; mientras que para la subescala de ansiedad una cantidad mayor o igual a cuatro (4) respuestas positivas indicaría un $50 \%$ de probabilidad de una alteración clínicamente significativa. A partir de los datos proporcionados se encontró que un $63 \%$ correspondiente a 72 cuidadores pudiesen padecer de depresión, un $37 \%$ correspondiente a 43 cuidadores fueron calificados como sin rasgos depresión, y al menos $52 \%$ correspondiente a 60 cuidadores pudiesen padecer de ansiedad, mientras que un 48 $\%$ correspondiente a 55 cuidadores fueron calificados sin rasgos ansiedad.

Tabla 2. Nivel de sobrecarga. $(\mathrm{n}=115)$

\begin{tabular}{|l|c|}
\hline Nivel de sobrecarga & Cantidad de personas \\
\hline No sobrecarga & $82(71 \%)$ \\
\hline Sobrecarga moderada & $14(12 \%)$ \\
\hline Sobrecarga severa & $19(17 \%)$ \\
\hline
\end{tabular}

Fuente: Ferreras et al. Internado Medicina Social, noviembre-diciembre 2019

Según la prueba de Zarit para sobrecarga, una cantidad menor a 46 puntos significa no sobrecarga, de 46 a 56 puntos refiere una sobrecarga moderada y mayor a 56 puntos hace referencia a una sobrecarga intensa. En este gráfico de barras se evalúa el nivel de sobrecarga de los cuidadores, para el cual se obtuvo que un $71 \%$, correspondiente a 82 cuidadores, no presentaron sobrecarga; el $17 \%$, 19 evaluados, presentaron una sobrecarga intensa; mientras que $12 \%$, igual a 14 personas, presentaron sobrecarga moderada.

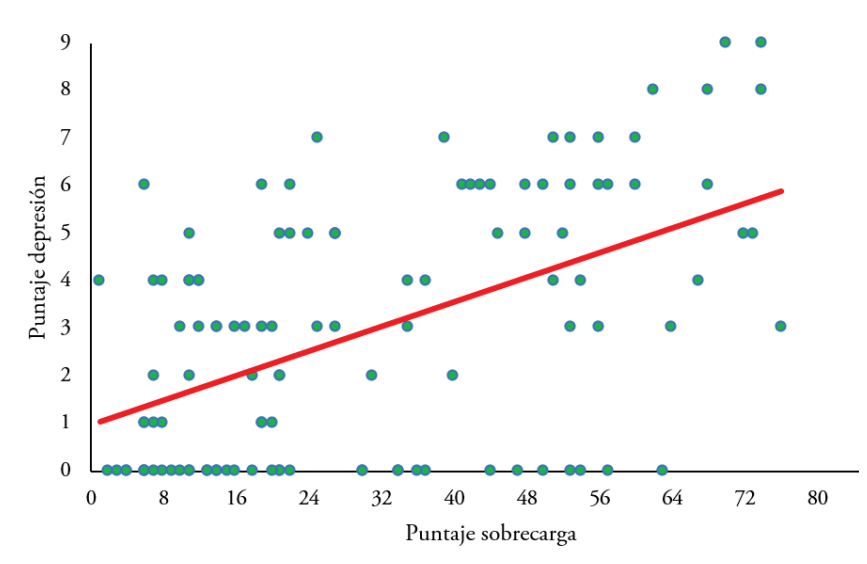

Figura 1. Gráfica de relación depresión con la sobrecarga $(n=115)$

Fuente: Ferreras et al. Internado Medicina Social, noviembre-diciembre 2019.

En esta gráfica se evalúa la relación del puntaje de sobrecarga dado por la prueba de Zarit con el puntaje de depresión dado por Goldberg en la subescala de depresión. Se ha encontrado una correlación positiva muy baja $\left(r^{2}=0.27\right)$. Se puede decir que por cada punto que aumente la sobrecarga, aumentará 0.065 en el puntaje de depresión. Sin embargo, como $\mathrm{r}^{2}$ es pequeño, la sobrecarga no es suficiente para explicar el comportamiento de la depresión, disminuyendo la certeza de la predicción.

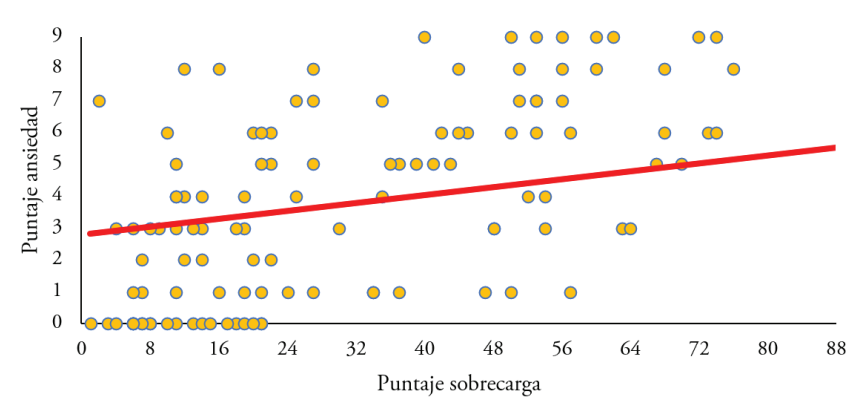

Figura 2. Gráfica de relación ansiedad con la sobrecarga $(\mathrm{n}=115)$

Fuente: Ferreras et al. Internado Medicina Social, noviembre-diciembre 2019
En esta gráfica se evalúa la relación del puntaje de sobrecarga, dado por la prueba de Zarit, con el puntaje de depresión dado por Goldberg en la subescala de ansiedad. Se ha encontrado una correlación positiva muy baja $\left(r^{2}=0.14\right)$. Se puede decir que por cada punto que aumente la sobrecarga, aumentará 0.031 en el puntaje de ansiedad. Sin embargo, como $\mathrm{r}^{2}$ es pequeño, la sobrecarga no es suficiente para explicar el comportamiento de la ansiedad, disminuyendo la certeza de la predicción.

Tabla 3. Edad y sexo $(\mathrm{n}=115)$

\begin{tabular}{|c|c|c|c|}
\hline & Femenino & Masculino & Total \\
\hline$<\mathbf{2 0}$ & $2(2 \%)$ & $0(0 \%)$ & $2(2 \%)$ \\
\hline $\mathbf{2 0 - 3 9}$ & $19(17 \%)$ & $6(5 \%)$ & $25(22 \%)$ \\
\hline $\mathbf{4 0 - 6 0}$ & $\mathbf{4 1}(\mathbf{3 6} \%)$ & $\mathbf{1 4}(\mathbf{1 2} \%)$ & $55(48 \%)$ \\
\hline$>\mathbf{6 0}$ & $27(23 \%)$ & $6(5 \%)$ & $33(28 \%)$ \\
\hline Total & $89(77 \%)$ & $26(23 \%)$ & $115(100 \%)$ \\
\hline
\end{tabular}

Fuente: Ferreras et al. Internado Medicina Social, noviembre-diciembre 2019

En la tabla se muestra la distribución de la muestra en función del sexo y edad, donde se encontró que el $77 \%$, correspondiente a 89 cuidadores, eran del sexo femenino mientras que el $23 \%$, constituido por 26 cuidadores, eran del sexo masculino. En cuanto a rangos de edad, el más frecuente fue de 40-60 años con $48 \%$, correspondiente a 55 cuidadores, seguido de $>60$ años con $28 \%$ igual a 33 individuos, el grupo 20-39 ańos con $22 \%$, equivalente a 25 personas, $\mathrm{y}<20$ años con $2 \%$, siendo 2 cuidadores.

\section{Discusiones y conclusiones}

De la muestra, un $63 \%$, correspondiente a 73 cuidadores, presentó una probable depresión. Este comportamiento concuerda con los establecidos en las literaturas, como en el artículo publicado por la Revista Cubana de Enfermería en el 2016 , donde demuestran que los cuidadores principales de pacientes con enfermedad de Alzheimer evidenciaron mayor malestar ante la presencia de agitación o agresión, alteraciones del sueño, trastornos de hábitos alimentarios, depresión, repeticiones, ansiedad, apatía e irritabilidad ${ }^{14}$.

Con respecto a la presencia de ansiedad, se encontró que un $52 \%$, correspondiente a 73 cuidadores, pudiese padecer de ansiedad, datos que concuerdan con la investigación citada anteriormente, donde uno de los síntomas más frecuentes fue la ansiedad, con un $22,85 \% 15,16$.

Con base en la prueba de Zarit para sobrecarga, se obtuvo que la mayor cantidad de cuidadores no presentaron ningún grado de sobrecarga, siendo este el $71 \%$, correspondiente a 82 cuidadores. En una investigación realizada en el 2012, por el departamento de Enfermería de la Universidad Austral de Chile, se determinó que de 67 cuidadores más de la mitad presentó sobrecarga intensa ${ }^{15}$. Esto demuestra diferencias marcadas en gran magnitud entre los grupos evaluados, en contraste con la ahora llevada a cabo, en el cual más de la mitad no presentó sobrecarga.

En la presente investigación se observó una correlación positiva muy baja en la subescala de depresión ( $\mathrm{r} 2=0.27$ ) y en la subescala de ansiedad ( $\mathrm{r} 2=0.14$ ). Lo cual concuerda nuevamente con los datos encontrados en la investigación realizada por la Universidad Austral de Chile, donde la depresión y la ansiedad obtuvieron un $22,85 \%{ }^{15}$, síntomas resultantes del grado de sobrecarga que estos presentan.

Sin embargo, varios estudios revisados ${ }^{1,15}$ mostraron asociación de la sobrecarga con variables como sexo, donde las féminas fueron la mayoría de la población que representa a los cuidadores hasta en un $82,8 \%{ }^{1}$. Correspondiendo con los resultados de la presente investigación, donde se demostró 
que un $76 \%$, compuesto por 87 cuidadores, era del sexo femenino. De la misma forma, la edad fue una variable que de igual manera coincidió con los datos previamente mencionados ${ }^{1,15}$, donde el $45 \%$ de los cuidadores se encontraron entre las edades de 41-60 ańos.

\section{Bibliografía}

1. Rodríguez M, Álvarez T, Martínez E, Valdivia $S$, Borroto I, Pedraza H. El síndrome del cuidador en cuidadores principales de ancianos con demencia Alzhéimer [Internet]. Scielo. Universidad de Ciencias Médicas de Sancti Spíritus; 2017. Available from: http://scielo. sld.cu/scielo.php?script=sci_arttext\&amp;pi$\mathrm{d}=$ S160889212017000100007\&amp;lng=es. [Cited 2019].

2. López M, Orueta R, Gómez-Caro S, Sánchez A, Carmona J, Alonso F. The caregiver burden and its repercussions on quality of life and health. Rev Clin Med Fam [Internet]. 2009; 2(7):332-9. Disponible en: http://scielo.isciii. es/scielo.php?script=sci_arttext\&pid=S16 99-695X2009000200004\&lng=e [citado 2019 Dec 24]

3. GBD 2015 Risk Factors Collaborators. Global, regional, and national comparative risk assessment of 79 behavioural, environmental and occupational, and metabolic risks or clusters of risks, 1990-2015: a systematic analysis for the Global Burden of Disease Study 2015 [published correction appearsinLancet.2017Jan7;389(10064):e1]. Lancet, 2016;388(10053):1659-724. Doi:10.10 16/S0140-6736(16)31679-8

4. Mejía N. Enfermedades crónicas no transmisibles [Internet]. 2015. Available from: https:// listindiario.com/la-vida/2015/03/16/359953/ enfermedades-cronicas-no-transmisibles [cited 2019 Dec 5]
5. Flores I, Montalvo A, Herrera A, Romero E. Afectación de los bienestares en cuidadores de niños y adultos con enfermedad crónica. Revista de Salud Pública [Internet]. 2010;12(5):754-64. Available from: https://www.scielosp.org/pdf/ rsap/2010.v12n5/754-764/es [cited 2019]

6. World Health Organization. Salud mental: fortalecer nuestra respuesta [Internet]. World Health Organization; 2018. Available from: https://www.who.int/es/news-room/fact-sheets/ detail/mental-health-strengthening-our-response [cited 2019].

7. Cabada E, Martínez V. Prevalencia del síndrome de sobrecarga y sintomatología ansiosa depresiva en el cuidador del adulto mayor. Revista Psicología y Salud [Internet]. 2017;27(1):53-9. Available from: https://psicologiaysalud.uv.mx/ index.php/psicysalud/article/viewFile/2436/ 4287 [cited 2019]

8. Tinker A. How to Improve Patient Outcomes for Chronic Diseases and Comorbidities [Internet]. Health Catalyst. 2017. Available from: www. healthcatalyst.com/wp-content/uploads/2014/ 04/How-to-Improve-Patient-Outcomes.pdf [cited 2019].

9. Pedraza AM, Rodríguez-Martínez CE, Acuña R. Validación inicial la escala Zarit para medir el nivel de sobrecarga de padres/cuidadores de niños asmáticos. Biomédica [Internet]. 2013;33(3). Available from: https://revistabiomedica.org/ index.php/biomedica/article/view/813/2292 [cited 2019].

10. Zarit SH. Relatives of the impaired elderly: correlates of feelings of burden. Gerontologist [Internet]. 1980. Available from: https:// bi.cibersam.es/busqueda-de-instrumentos/ ficha?Id=96 [cited 2019]
11. Escala de Ansiedad y Depresión de Goldberg (EADG) [Internet]. www3.gobiernodecanarias.org. Gobierno de Canarias. Available from: https://www3.gobiernodecanarias.org/ sanidad/scs/content/d4256655-0f04-11 de9de1-998efb13096d/S_MENTAL8.pdf-*9 [cited 2019].

12. Goldberg DP, Hillier VF. A scaled version of the General Health Questionnaire. Psychol Med, 1979;9(1):39-145. Doi: 10.1017/s003 3291700021644. [Cited 2019].

13. La Encuesta de Discapacidad, Autonomía personal y situaciones de Dependencia: Cuestionario de Cuidadores/as principales. Instituto Nacional de Estadística de España [Internet]. Available from: https://www.ine.es/daco/daco42/ discapa/edad_cui.pdf [cited 2019]

14. Garzón M, Pascual Y, Collazo C. Psychological distress in main caregivers of Alzheimer's disease patients [Internet]. Revista Cubana de Enfermería. 2018;34(3):591-9. Available from: http://scielo.sld.cu/pdf/enf/v34n3/1561-2961enf-34-03-e1052.pdf
15. FloresE, RivasE, Seguel F. Nivel desobrecargaen el desempeńo del rol del cuidador familiar de adulto mayor con dependencia severa. Cienc. enferm. [Internet]. 2012;18(1): 29-41. Disponible en: https://scielo.conicyt.cl/scielo.php?script=sci arttext\&pid=S0717-95532012000100004\&ln$\mathrm{g}=$ es. http://dx.doi.org/10.4067/S0717-95532 012000100004 [citado 2019 Dic 07]

16. Bello J. Sobrecarga del cuidador primario de pacientes con enfermedades crónicas degeneraivas [Internet]. https://www.uv.mx/. Instituto Mexicano del Seguro Social; 2014. Available from: https://www.uv.mx/blogs/favem2014/files/ 2014/06/Tesis-gabriela.pdf [cited 2019]. 